\title{
INL Review of Fueling Machine Inspection Tool Development Proposal
}

George W. Griffith

March 2015

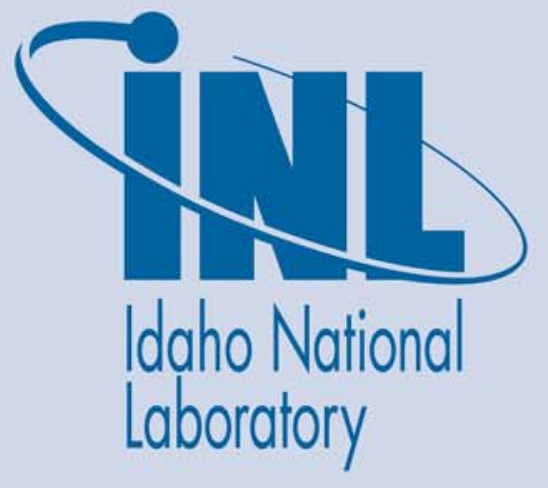

The INL is a U.S. Department of Energy National Laboratory operated by Battelle Energy Alliance 
INL/EXT-15-34730

\title{
INL Review of Fueling Machine Inspection Tool Development Proposal
}

George W. Griffith

March 2015

\author{
Idaho National Laboratory \\ Idaho Falls, Idaho 83415
}

http://www.inl.gov

Prepared for the

U.S. Department of Energy

Office of Nuclear Energy

Under DOE Idaho Operations Office

Contract DE-AC07-05ID14517 


\section{DISCLAIMER}

This information was prepared as an account of work sponsored by an agency of the U.S. Government. Neither the U.S. Government nor any agency thereof, nor any of their employees, makes any warranty, expressed or implied, or assumes any legal liability or responsibility for the accuracy, completeness, or usefulness, of any information, apparatus, product, or process disclosed, or represents that its use would not infringe privately owned rights. References herein to any specific commercial product, process, or service by trade name, trade mark, manufacturer, or otherwise, does not necessarily constitute or imply its endorsement, recommendation, or favoring by the U.S. Government or any agency thereof. The views and opinions of authors expressed herein do not necessarily state or reflect those of the U.S. Government or any agency thereof. 


\section{INL Review of Fueling Machine Inspection Tool Development Proposal \\ PURPOSE}

This paper provides a technical review of the AMEC Nuclear UK design report produced for EDF provided by James Fisher Nuclear Ltd (JFN). The design proposes a visual inspection system for the fueling machine inspection tool utilized at the Advanced Gas-Cooled Reactors (AGRs). The review is limited to the design proposal and information provided in the AMEC report given to Idaho National Laboratory.

\section{Introduction}

The AGRs are dual unit, $2 \mathrm{x}(1,623 \mathrm{MWth} / 660 \mathrm{MWe}) \mathrm{CO}_{2}$-cooled reactors. The reactors use graphite as a moderator (graphite structures are shown in Figure 1). The reactors started operation between the late 1970s and 1980s.i The long period of thermal cycling and exposure to radiation during operation has affected the graphite moderator structure. As part of improving the inspection of the graphite structures, a visual inspection system is desired to supplement existing physical measurements and eddy current techniques, in order to better evaluate the condition of the graphite blocks that make up the reactor. Retrieved data will be used to underpin the extended operations of the AGR reactors.

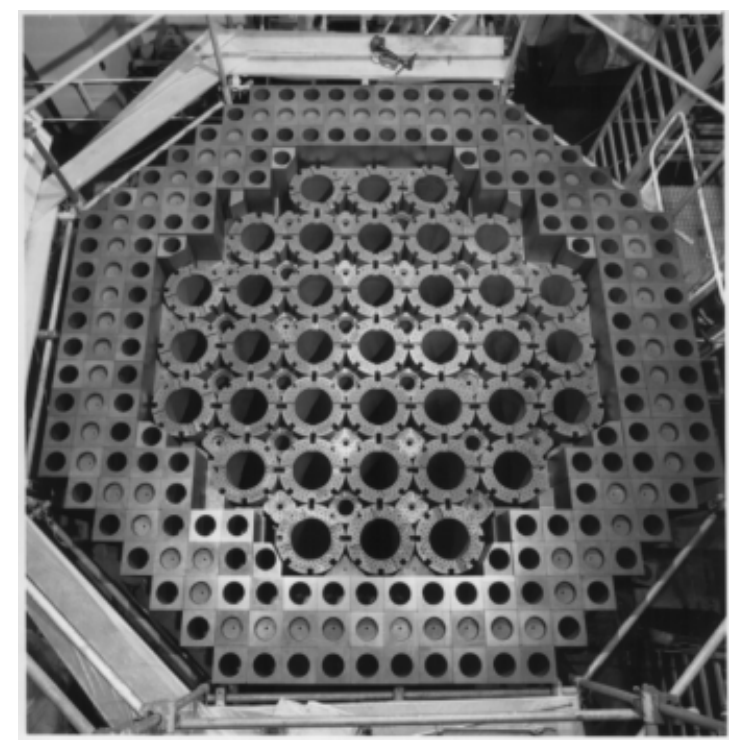

Figure 1. Approximate AGR graphite moderator and internal structure.

A visual inspection within the pressurized core of a fueled nuclear reactor, even at zero power, is a challenge because of the elevated temperatures, cooling gas flows, high radiation field created by the exposed fuel and attendant nuclear fission products. Ideally, the graphite blocks would be efficiently inspected while the reactor is off load, at or near operating pressurized conditions. Adding this flexibility greatly complicates the inspection tool design because of increased pressure and temperature and greatly increased radiation.

The optical design proposed in the AMEC report is influenced by the need to operate and survive in a pressurized, offload reactor core. The proposed basic design appears capable of achieving acceptable inspection results, even in the harsh conditions of a low-power operating AGR. Some modification to the AMEC design features and refinement can provide better operational performance. 


\section{RECOMMENDATIONS}

The following are recommendations regarding the design of the AMEC bench tested Light Pipe design to enable potential incorporation into a Fueling Machine Inspection Tool (FMIT):

1. The design of the viewing tube should be changed from a vacuum tube to a backfilled helium atmosphere. A helium atmosphere will minimize optical distortion compared to any other practical atmosphere. Continually backfilling the helium would further reduce optical distortion, prevent debris from entering the tube and keep the atmosphere helium.

a) Elimination of the vacuum tube, with a helium atmosphere, prevents the need for developing a vacuum system safety margin calculation that is capable of accounting for all the operating and safely margins. An atmospheric tube eliminates the sensitivity of the tube to dimensional imperfections, radiation damage, dents and scratches, and window sealing that would add confidence and simplicity to the design.

b) Eliminating the vacuum tube design also eliminates the potential of having the tube collapse while in service. The potential for bent and distorted metal after a collapse that could create significant engineering and operational challenges to remove. Evaluation of any resulting damage to the vessel internals would also be difficult making the cost of failure high.

2. Perform a more detailed analysis of the inspection tube's stresses, including the current stress analysis, a tube collapse analysis, and a cantilever stress analysis. The detailed analysis will help define a needed safety margin and help ensure the tube is robust enough for operational efficiency and safety.

3. If practical, replace stainless steel in the tube design with a zirconium alloy. Stainless steel has a large neutron thermal neutron absorption cross section and will act as a control rod when the reactor is operating. The zirconium, with a lower thermal neutron absorption cross section, will not have the same effect on the reactor at low power, making operations easier. This is only viable if the tube support structure is already a low absorption material like zirconium.

\section{DESIGN DESCRIPTION}

The basic system is based on a digital camera fitted with a lens, allowing it to act as a telescope to the end of the $20 \mathrm{~m}$ inspection tube. The bottom of the tube has a mirror to allow viewing of the graphite bricks through a window in the side of the inspection tube. The mirror image digitally recorded by the camera is unfolded by software, yielding a conventional image to be analyzed.

Placing the camera at the top of the tube helps avoid the majority of the radiation and heat from the reactor. This is required to avoid an extremely short life of the camera or transmission optics.

\section{POSITIVE FEATURES \\ 4.1 Optics}

The optical system at this early stage of development appears to work well for the small desk top rig, scaled up bench top rig and full length test rig. Use of a digital camera and a lens with a long focal length that acts as an inspection telescope worked well. Use of a finely finished stainless steel mirror was a positive feature. Optimizing the camera to lighting conditions and window/mirror geometry appear to offer additional improvements. The robustness and simplicity of the optical system will provide operational benefits. 


\subsection{Software}

The simple software solution demonstrated good results. The library transform software, AForge.net, did a good job of unfolding the conical image. The mirror and image collection appeared to be limiting the image quality more than the unfolding software.

Using the encoder data to allow fixed segments to be stitched together was a successful approach and should be successful in the full-sized system. Image enhancement of the crack image indicates some of the benefits of using digital cameras. Use of filters allowed cracks to be detected even when the unprocessed image did not provide initial evidence.

\section{Lighting Demonstration}

The lighting system demonstrated successful performance for the three test configurations: table top rig, bench top rig and full-length rig assembled at AMEC. The lighting system and requirements is evaluated in INL report INL/EXT-15-35074. A very long light pipe may be excessively heavy or susceptible to darkening. Placing electronic lights close to the mirror would expose them to excess radiation and heat damage. LED lights near the camera show the potential to avoid these problems and provide acceptable lighting.

6. CHALLENGES

\subsection{Vacuum Tube Design}

In the design, a vacuum tube contains the optical system. The vacuum is intended to eliminate optical distortion caused by variations in the index of refraction in the tube. Index of refraction varies with density, temperature, and composition. The simplest option would be to include the $\mathrm{CO}_{2}$ atmosphere because $\mathrm{CO}_{2}$ has one of the highest indexes of refraction (see Table 1). However, this option will tend to add to the optical distortion and may allow reactor coolant into the tube, causing contamination.

Replacing the $\mathrm{CO}_{2}$ with helium would reduce the optical distortion effect significantly. The overall effect of the index of refraction will tend be reduced as the system temperature goes up and the density of the fill gas goes down. ${ }^{\text {ii }}$

Table 1. Index of refraction for common gases.

\begin{tabular}{lc}
\hline \multicolumn{1}{c}{ Gas } & $\begin{array}{c}\text { Index of Refraction } \\
\text { (sodium D line, 101 KPa, 0 }{ }^{\circ} \mathrm{C} \text { ) }\end{array}$ \\
\hline Helium & 1.000035 \\
Air & 1.000292 \\
Nitrogen & 1.000298 \\
$\mathrm{CO}_{2}$ & 1.000449 \\
Hydrogen & 1.000132 \\
\hline
\end{tabular}

Additional improvements could be made by continually purging the optical tube. This would even out the temperature differences. Creating a more uniform radial temperature difference will reduce optical variations. Purging may also add turbulence, depending on geometry and flow rate which affect the Reynolds number, which would average out the overall index of refraction and further reduce the optical distortion.

Using a tube with a gas atmosphere would provide internal pressure to balance the outer reactor pressure. This will eliminate the risk of vacuum tube collapse and buckling. Including an atmosphere also prevents inward leaks from being a critical failure. Eliminating the potential for tube collapse also 
eliminates the chance of creating a distorted and bent structure inside the core. Tube support structure details potentially affect the damage risk.

\subsection{Buckling Analysis}

The design of a vacuum vessel with a length-over-diameter ratio of approximately 275 creates some difficulties. The tube will be under multiple operating stresses from mechanical loading, operational movement (and contact), thermal gradients, and radiation-induced changes. With a long narrow tube being loaded and unloaded from the core, multiple complex stresses will occur. Any or all of these induced loads can introduce damage and distortion. The inevitable damage would make buckling and collapse much more likely due to the nonlinear behavior in buckling. ${ }^{\text {iii }}$

The analysis of buckling referenced in the report is a straight forward take on buckling that accounts for approximations utilizing a safety factor. The safety factor utilized at CERN for largely static structures is likely too low to be adequate for an AGR inspection tool. ${ }^{\text {iv }}$ Additional collapse modes exist, where the structure collapses radially. ${ }_{\text {iiii }}$ These failure modes should be considered.

More complex analyses that account for damage and irregularities are recommended if a significantly higher safety factor is not implemented. ${ }^{\mathrm{v}}$ These analyses should include non-circular assumptions, point defects (dents), or scratches. A detailed analysis using modern analysis tools is strongly suggested to evaluate the complex stress field.

The buckling assumptions in operation are potentially affected by the details of the structure used to support the tube. A tube supported from the top and suspended would be under less compressive stress than a tube supported from below. The need to manage external pressure can be eliminated by using a helium atmosphere (as noted in Section 5.1.1).

Additional, but more conventional, stress can be created during handling. The length over which the tube can be cantilevered would be useful information. The tube will undergo stress as it is fabricated, shipped, handled onsite and installed in the Fuel Fusion Materials Irradiation Test Facility. These handling stresses will need to be managed or included in the design requirement.

\subsection{Stainless Steel}

\section{Material Issues}

Stainless steel is prone to various radiation-induced material changes. Stainless steel embrittles in neutron fields due to neutron damage that displaces atoms in the steel and generates helium. Even though the total dose is unlikely to be beyond the capabilities of stainless steel, embrittlement and the thin tube thickness may make the tube susceptible to damage, causing leaks into the tube. It is suggested that zirconium tubing be considered for a non-vacuum tube application.

\section{Reactivity}

An anticipated initial application for the inspection tool is use during pressurized, zero power operation, although ultimately low power operations may be considered. The long inspection tube will be placed at variable positions within the core. The tube will act as a control rod in the core. Stainless steel is an absorber of neutrons. Zirconium has much lower thermal neutron absorption than stainless steel. To reduce the operational power effects and make the tool easier to use, it is again suggested that zirconium aluminum be used for the tube. Aluminum would be an acceptable choice; however, it is not capable of operating at the $500^{\circ} \mathrm{C}$ temperature that is anticipated at low power.

It is currently assumed that the fuel machine inspection tool will be made of a low absorption material, like zirconium, to minimize interference with power operations. If the current inspection tool is largely steel, then there is little need for the optical tool to be zirconium. 


\section{Bellows}

A bellows will be needed to account for changes in operating temperatures and pressures as the inspection tool is used. Consideration of where the bellows is positioned and what type of mechanical flexibility or adaptability is required to avoid unwanted optical changes will be desirable.

\subsection{Optics}

The mirror and window structure at the bottom of the tube remain a design challenge. The mirror and window will need to be fabricated from materials that do not darken when exposed to high radiation fields and high temperature.

Illuminating the mirror/graphite at the end of a $20 \mathrm{~m}$ tube is also a challenge. The current solution that uses a light pipe may or may not be acceptable, depending on the details of the design. A separate review of the lighting options was prepared by Idaho National Laboratory in INL/EXT-15-35074.

\section{Digital Camera}

The proposed design still requires the incorporation of an onboard Digital Camera. Even if this device is located at the upper end of the FMIT, it will experience elevated temperature / radiation environmental conditions which will exist in the Standpipe (above the reactor core) and while stored within the Fuelling Machine turret, adjacent to an irradiated stringer.

${ }^{\text {i }}$ Erik Nonbel, "Description of the Advanced Gas Cooled Type of Reactor (AGR)," National Laboratory Roskilde, Denmark, November 1996.

${ }^{\text {ii }}$ NIST Physical Measurement Laboratory, Engineering Metrology Toolbox, http://emtoolbox.nist.gov/Wavelength/Documentation.asp\#CommentsRegardingtheCalculations, web page visited 5/19/2015

iii C. de Paor, D. Kelliher, K. Cronin, W. M. D. Wright, and S. G. McSweeney, "Prediction of vacuum-induced buckling pressures of thin-walled cylinders," Thin-Walled Structures, 55: 1-10, Elsevier, March 2012.

${ }^{\text {iv }}$ C. Hauviller, "Design Rules for Vacuum Chambers," CERN, Geneva, Switzerland, 2007

v External Pressure, "Pressure Vessel," http://www.pveng.com/ASME/ASMEComment/ExternalPressure/ExternalPressure.php, web page visited 3/17/2015. 